Bundesgesundheitsbl - Gesundheitsforsch Gesundheitsschutz 2005 · 48:951-953

DOI 10.1007/s00103-005-1103-9

(C) Springer Medizin Verlag 2005
G. Dettweiler -W. Weigelt · Robert Koch-Institut, Berlin

\section{Der Weg zum neuen Internetauftritt des Robert Koch-Instituts}

\section{Ein Projektbericht}

\section{Erfolg und Bedarf für Neues}

Der Internetauftritt des Robert Koch-Instituts (RKI) hat sich im Verlauf seines relativ kurzen Daseins zu einem unverzichtbaren Arbeitsinstrument und zu einer Erfolgsgeschichte für das Institut entwickelt. Gestartet 1997 mit einigen Hundert Seiten im World Wide Web, war der Inhalt des elektronischen Mediums im Jahr 2000 bereits auf 4000 Seiten angewachsen, im Jahr 2004 hatten sich die Seitenzahlen nochmals auf 8000 verdoppelt. $\mathrm{Pa}-$ rallel dazu stieg auch die Zahl der Seitenaufrufe. Nach kontinuierlichem Anstieg seit 1997 wurden im November 2002 erstmals mehr als eine Million Seitenaufrufe pro Monat registriert, im März 2003 wurde bereits die Marke der 2 Millionen erreicht (• Abb. 1).

Hinter diesen Zahlen steht die Arbeit vieler Mitarbeiter des Instituts, zunächst natürlich der Mitarbeiter aus Wissenschaft und Administration, die für die Inhalte sorgen. Die technische Betreuung oblag dem IT-Referat, die Umsetzung der Vorlagen ins Netz nahmen Mitarbeiter mit Kenntnissen als HTML-Editoren vor, deren Zahl seit dem Jahr 2000 von 12 auf 22 gestiegen war. Das Referat Presse/ Öffentlichkeitsarbeit/Bibliotheken betreute eine Reihe von Inhalten auf den Seiten und achtete darauf, dass das Erscheinungsbild dem Corporate Design des Instituts entsprach und vergleichbare Bereiche einheitlich dargestellt wurden. Hierfür wurde im Jahr 2001 die Position des Internet- beauftragten geschaffen, der als Ansprechpartner auch die Weiterentwicklung des Internetauftritts koordiniert.

Durch das Wachstum des Internetauftritts und die damit verbundenen steigenden Anforderungen und Aufwände haben sich aber auch Probleme eingestellt. So wurde es z. B. zunehmend schwieriger, ohne ein modernes Redaktionssystem neue Inhalte ausreichend rasch im Internet zu publizieren. Ein Engpass bestand darin, dass nur wenige Mitarbeiter Kenntnisse in der Erstellung von HTMLSeiten besitzen, die notwendig sind, um Internetseiten zu erstellen. Die Vorgabe und Einhaltung von Standards gestaltete sich bei der wachsenden Zahl der Ak- teure problematisch, da die eingesetzte Technik Standardisierungen nur sehr eingeschränkt unterstützte. Dies führte u. a. dazu, dass sich einige inhaltliche Bereiche sehr heterogen und zu wenig strukturiert entwickelten mit der Folge leidender Übersichtlichkeit und Defiziten bei der Nutzerführung. Auch das Layout der Seiten hatte dem Zeitgeist Tribut zu zollen und war nicht mehr auf dem aktuellen Stand moderner Internetseiten. Stichworte sind hier die Hauptnavigation am Ende der Site, keine Anwendung der Marginalspalte, Mängel beim Identitäts- und fehlender Bildbereich. Dennoch waren die Rückmeldungen von Nutzern zum Layout des alten Auftritts des RKI bis zuletzt überwiegend

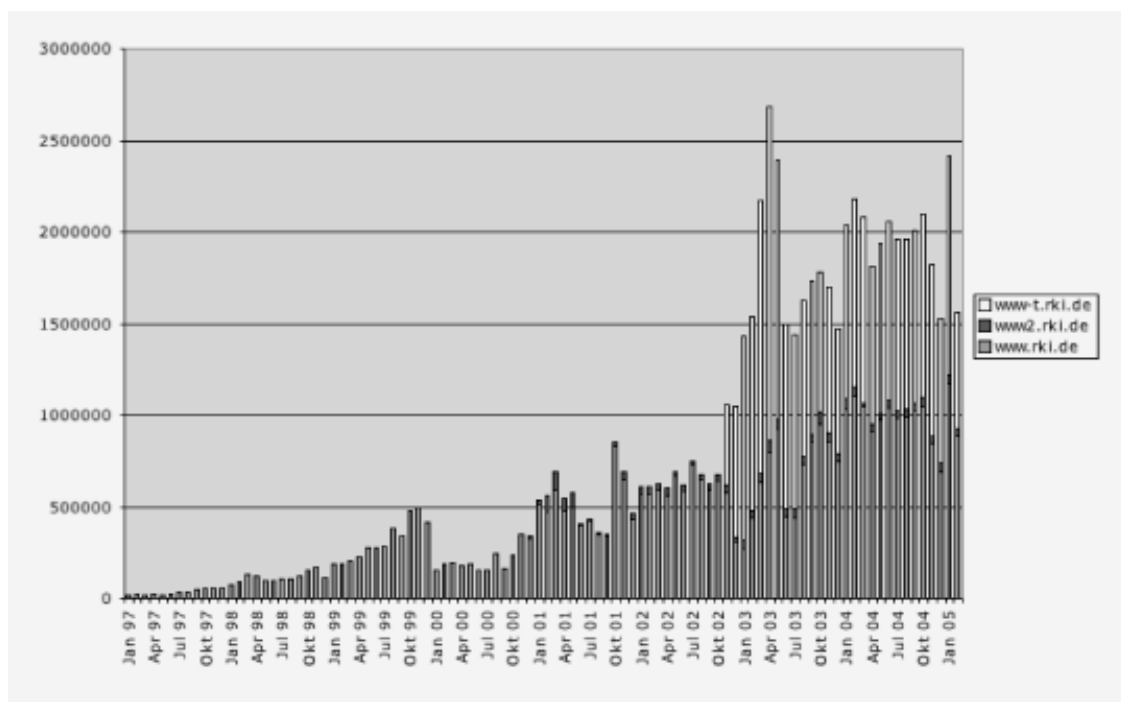

Abb. $1 \triangle$ Zahl der Aufrufe der Internetseiten des Robert Koch-Institutes (RKI) pro Monat seit 1997 
positiv. Gelobt wurde insbesondere, dass er sich auf die Inhalte konzentrierte und auf neuere Anwendungen wie Pop-ups, Laufbänder, Animationen völlig und auf Bilder weitgehend verzichtete. Dies wurde auch bei den neuen Seiten beibehalten.

Auslöser für das RKI, seinen Internetauftritt zu überarbeiten, war die Vorschrift, dass die Internetseiten der Institutionen der Bundesverwaltung bis zum 31.12.2005 barrierefrei zu gestalten sind. Die diesbezüglichen Anforderungen regelt die Verordnung zur Schaffung barrierefreier Informationstechnik nach dem Behindertengleichstellungsgesetz (BITV) vom 17. Juli 2002. Ziel der Verordnung ist es, behinderten Menschen zu erleichtern, die Schlüsseltechnologien des Internets zu nutzen. Die in der BITV formulierten Anforderungen konzentrieren sich darauf, Hilfestellungen bei verschiedenen Ausprägungen von Sehstörungen zu geben. Diese betreffen z. B. Vorgaben zu den Schriftarten und Schriftgrößen, die Verwendung ausreichender Farbkontraste und Vermeidung bestimmter Farbkombinationen bis hin zu Auszeichnungen und Erläuterungen über die dargestellten Inhalte und Bilder. Ein wesentliches Ziel der Verordnung ist es, dass spezielle Programme, so genannte Screen Reader, die Seiten vorlesen können. Dies setzt grundsätzlich voraus, dass Inhalt und Layout eines Internetauftritts getrennt sind und dass der Inhalt von Internetseiten nicht durch Tabellen formatiert wird. Letzteres war bei den Internetseiten des RKI der Fall, sodass man vor der Alternative stand, entweder die Seiten durch die Mitarbeiter des RKI umzuarbeiten oder den Internetauftritt auf ein System zu migrieren, das die Voraussetzungen der Verordnung erfüllt.

\section{Das Projekt}

Es wurde entschieden, den Internetauftritt des RKI auf ein Content-Management-System (CMS) zu migrieren. Maßgeblich für diese Entscheidung war zum einen, dass eine vom RKI durchgeführte Wirtschaftlichkeitsbetrachtung (Wibe 21) dies als die kostengünstigere Alternative gegenüber der Umarbeitung der bestehenden Internetseiten auf dem alten System ergeben hatte. Darüber hinaus erleichtern Content-Management-Systeme, Internetseiten zu erstellen und zu pflegen. Die Wahl des RKI fiel auf ein CMS, das als Basiskomponente im Rahmen der Initiative der Bundesregierung „BundOnline“ für die Institutionen des Bundes entwickelt worden war, den „Government Site Builder“.

Diese Wahl bot dem RKI eine Reihe von Vorteilen:

- Unterstützung bei der Planung der Einführung des CMS durch Beratung, Leitfäden, Toolboxen,

- Unterstützung bei der Erstellung von Konzepten für den Neuauftritt,

- Synergieeffekte bei der Einführung durch die gemeinsame Nutzung des Produktes von verschiedenen Einrichtungen des Bundes,

- Erfahrungsaustausch der Anwender,

- Übernahme eines grundsätzlich einheitlichen Seitenaufbaus für Internetauftritte des Bundes (Internet Styleguide des Bundes) mit der Möglichkeit individueller Layout-Anpassung,

- Möglichkeiten zur Mitbestimmung bei der Weiterentwicklung des Produktes,

- günstige Lizenzkosten.

Nachdem die grundsätzliche Entscheidung für das CMS gefallen war, wurde im RKI mit einem Workshop im Dezember 2003, an dem die Leitung, die Abteilungsleitungen, der Personalrat und externe Berater teilnahmen, dessen Einführung als Projekt initiiert. Als Projektlaufzeit wurde ein Jahr festgesetzt, 2 Personen wurde die Projektleitung übertragen und ein 9-köpfiger Beirat berufen, der die fachlichen Organisationseinheiten des RKI im Projekt vertrat. Der Beirat hatte dabei die Aufgabe, die Anforderungen an den neuen Internetauftritt mit den Abteilungen abzustimmen.

Der aufgestellte Projektplan sah im zeitlichen Ablauf folgende größere Projektschritte vor: Erstellung von Anforderungskonzept, Redaktionskonzept, Content Modell (Dokumententypen), Navigationskonzept, Layout, anschließend Vorbereitung und Migration der vorhandenen Inhalte auf das CMS, Probebetrieb des CMS, Qualitätssicherung, Freischaltung

${ }^{1}$ Zur Initiative BundOnline der Bundesregierung siehe http://www.wmsbundonline.de/ des neuen Auftritts. Die Arbeiten zur Ermittlung der benötigten Dokumententypen für den neuen Auftritt und für die Migration der vorhandenen Inhalte auf das neue System wurden an externe Auftragnehmer vergeben.

\section{Die Konzeptphase}

Im Anforderungskonzept wurden die grundsätzlichen Bedürfnisse des RKI an den Leistungsumfang des neuen Internetauftritts formuliert und mit der Leistungsbeschreibung des CMS abgeglichen. Wesentliche Punkte waren dabei die Ausfallsicherheit des Systems, eine Verfügbarkeit 7 Tage/24 Stunden, kurze Publikationszeiten, Mehrsprachigkeit, die Verfügbarkeit geschützter Bereiche, einer Expertensuche, einer Sitemap, von Kontaktformularen, die automatische Generierung einer Liste neuer Inhalte im Internet, eine Glossarfunktion, Zugriffsauswertung, Newsletter und die Möglichkeit, Drittsysteme, insbesondere Datenbanken, einzubinden. Im Anforderungskonzept wurde auch die Entscheidung getroffen, den Rechnerbetrieb (Hosting) nicht mehr am RKI, sondern am Bundesverwaltungsamt zu führen.

Die Diskussionen zum Redaktionskonzept ergaben einen 6-Augen-Workflow, um zukünftig Dokumente im Internet zu publizieren. Die Lokalredakteure erstellen die Dokumente, die Fachredakteure sind für die Qualitätssicherung der Dokumente verantwortlich, eine Redaktionsgruppe prüft formale Anforderungen wie die Verwendung der korrekten Formate sowie die Zuordnung neuer Inhalte zu den Navigationsknoten und publiziert die Dokumente. Insgesamt wurden 100 Redakteure für die Publikation von Dokumenten im Internet benannt. Mit dem CMS werden ausschließlich Dokumente publiziert, die bereits im Vorfeld abgestimmt worden sind, das CMS wird nicht als Vorgangsbearbeitungssystem eingesetzt.

Die Analyse des bestehenden Internetauftritts für das Content Modell ergab, dass das RKI 18 der mehr als 100 vorliegenden Dokumententypen (Templates) des CMS benötigt, um seine Inhalte dort abzulegen. Einige der Templates mussten für die Bedürfnisse des RKI angepasst, ein Template zusätzlich erstellt werden. 
Die Durchsicht der 8000 Internetseiten und die Ermittlung der notwendigen Templates wurde in Form eines 2-monatigen Werkvertrags an eine Auftragnehmerin vergeben.

Die Beratungen zum Navigationskonzept ergaben, dass die bestehende Navigationsstruktur der Internetseiten überarbeitet werden musste. Im Hauptmenü der deutschen Seiten wurden die bereits bestehenden Punkte „Das Institut“ und „Forschung “ weitergeführt mit erheblich erweitertem Inhalt der Forschungsseiten. Die Bereiche „Aktuelles“ und „Service“ wurden zu einem Punkt zusammengefasst. Der bisherige Hauptnavigationspunkt „Gesundheit und Krankheiten“ wurde aufgelöst, die Inhalte wurden besser strukturiert und neuen Oberbegriffen zugeordnet. Teile mit Daten zum Infektionsgeschehen und mit Maßnahmen zum Schutz vor Erregern wurden unter dem neuen Menüpunkt „Infektionsschutz“ subsumiert. Ergänzt wird diese Darstellung durch Informationen zu einzelnen Erregern unter „Infektionskrankheiten A-Z“. Neu ist der Bereich „Gesundheit A-Z“, der Studien und Arbeitsergebnisse zu häufig nachgefragten Gesundheitsthemen bündelt. Diese Rubrik ergänzt die umfangreichen Informationen der Internetseiten zur „Gesundheitsberichterstattung und Epidemiologie“. Hier werden die Arbeiten der Surveys und Sentinels des RKI vorgestellt, Daten und Berichte präsentiert und die angewandten epidemiologischen Methoden erläutert. Eingebunden in diese Seiten sind auch die Informationen aus der Dachdokumentation Krebs und über den derzeit laufenden Kinder- und Jugendsurvey, der erstmalig umfassend den Gesundheitszustand von Kindern und Jugendlichen im Alter von o-18 Jahren für ganz Deutschland untersucht.

\section{Implementierung des Content-Management-Systems}

Die Konzeptphase des Projektes wurde im August 2004 mit der siebten und letzten Sitzung des Projektbeirates abgeschlossen. Wie häufig bei Projekten ließ sich auch in diesem Fall der zu Beginn aufgestellte zeitliche Projektplan nicht immer einhalten. Während das Content Modell und das Redaktionskonzept planmäßig im Mai und im Juni 2004 vorlagen, zogen sich die Arbeiten am Navigationskonzept, die ursprünglich im April beendet sein sollten, bis Juli, die Arbeiten am Anforderungskonzept, die im März abgeschlossen sein sollten, bis August 2004 hin. Im September konnten dann mit einer Firma, die im Auftrag des Bundesverwaltungsamtes die Einrichtung des CMS realisierte, Beratungen zur Umsetzung der Vorgaben des RKI begonnen werden. In einem 4tägigen Workshop wurden die Anpassungen des Standardmandanten des Government-Site-Builders festgelegt, auf deren Basis dann die Verträge zur Umsetzung geschlossen wurden. Im Oktober 2004 wurde der Prototyp des neuen CMS am RKI installiert, bis November 2004 wurde der Prototyp an die festgelegten Spezifikationen angepasst und anschließend am Bundesverwaltungsamt eingespielt.

Damit begannen die Phasen der Schulungen für das CMS und der Übertragung von 8000 Seiten Inhalt des bestehenden Internetauftritts (Migration) auf das CMS. Die Schulungen von Mitarbeitern des RKI als Site-Administratoren und als Redakteure wurden am RKI durchgeführt. Die Migration der Inhalte wurde als Werkvertrag an 8 Studenten vergeben. Nach eintägiger Einweisung der Studenten in den Editoren des CMS wurden die einzelnen Bereiche des Internetauftritts an Teams zu je 2 Personen zur Migration aufgeteilt. Ein Student hatte die Aufgabe, die Migrationsarbeiten zu koordinieren und den Verlauf zu überwachen, die Site-Administratoren waren vor Ort, um technische und organisatorische Fragen unmittelbar zu klären. Begonnen wurde mit Dokumenten, die einheitlich strukturiert waren, wie z. B. mit der Publikationsliste und den Pressemitteilungen, um Erfahrungen mit dem neuen System zu sammeln. Daran schloss sich die Migration heterogener Bereiche an, abschließend wurden die internen Verlinkungen angepasst und die externen Links in das System eingepflegt. Diese Arbeiten waren im Dezember 2004 abgeschlossen. In den 6 Wochen der Migration waren mehr als 10.000 Dokumente im CMS erstellt worden.

Im nächsten Schritt wurden die Arbeiten zur Qualitätssicherung aufgenommen. Nach der Migration wurde nun erstmals das vollständige Ergebnis der bisherigen konzeptionellen, redaktionellen und technischen Arbeiten am Bildschirm sichtbar. Es zeigte sich, dass z. B. am Layout noch Verbesserungen notwendig waren, sowohl am RKI-Mandanten des Government-Site-Builders als auch an verschiedenen Templates, die zur Darstellung von Texten, Listen und Tabellen definiert worden waren. Eine weitere Firma wurde beauftragt, Layoutentwürfe mit Darstellungsvarianten für die Haupt- und Subnavigation, den Bildbereich, den Contentbereich und die Marginalspalte zu erarbeiten. Für die Einspielung der verschiedenen Änderungen des Layouts im CMS, die von der Leitung des RKI beschlossen worden waren, war ein weiterer Supportvertrag notwendig. Auch die Vollständigkeit und Darstellung der Inhalte musste vor der Freischaltung nochmals überprüft werden. Da eine Reihe Mitarbeiter des RKI als Redakteure geschult worden waren, konnten diese Arbeiten auf viele Schultern verteilt werden. Dies war auch notwendig, da nach der Migration doch noch ein erheblicher Bedarf für Ergänzungen bei Inhalten und für Korrekturen an der Darstellung der Texte und bei der Pflege von Verlinkungen bestand.

Als Termin, an dem die Prüf- und Pflegearbeiten abgeschlossen sein mussten, wurde der 11. Februar 2005 festgelegt. Danach war noch eine Woche Zeit, in der u. a. am Bundesverwaltungsamt die letzten geänderten Inhalte vom Rechner, auf dem die Redakteure arbeiten, auf den Life-Server überspielt, ein geschützter Testzugriff und der Redirect auf die Server am Bundesverwaltungsamt eingerichtet werden konnten. Am 18. Februar 2005 wurde der neue Internetauftritt freigeschaltet.

\section{Korrespondierender Autor G. Dettweiler}

Robert Koch-Institut, Nordufer 20, 13353 Berlin E-Mail: presse@rki.de 